\title{
Alternate oscillations in semiconductor ring lasers
}

\author{
M. Sorel and P. J. R. Laybourn \\ Department of Electronics and Electrical Engineering, University of Glasgow, Oakfield Avenue, Glasgow G12 8LT, Scotland, UK
}

A. Scirè and S. Balle

Instituto Mediterráneo de Estudios Avanzados, Consejo Superior de Investigaciones Cientificas-Universidad de las Islas Baleares, Campus Universidad de las Islas Balears, E-07071 Palma de Mallorca, Spain

\author{
G. Giuliani, R. Miglierina, and S. Donati
}

Dipartimento di Elettronica, Università di Pavia, Via Ferrata 1, I-2710o Pavia, Italy

Received June 3, 2002

\begin{abstract}
We report on fabrication and characterization of single-longitudinal- and transverse-mode semiconductor ring lasers. A bifurcation from bidirectional stable operation to a regime with alternate oscillations of the counterpropagating modes was observed experimentally and is theoretically explained through a two-mode model. Analytical expressions for the onset and the frequency of the oscillations are derived, and $L-I$ curves numerically evaluated. Good quantitative agreement between theory and measurements made over a large number of tested devices is obtained. (C) 2002 Optical Society of America

OCIS codes: $140.3560,230.3120,190.3100$.
\end{abstract}

The two-mode dynamics in ring laser systems has been the subject of a large number of experimental and theoretical investigations. ${ }^{1}$ From the fundamental point of view, analysis of the peculiar symmetry properties of the ring laser provides insight for nonlinear dynamics studies, ${ }^{2}$ enhancing the knowledge of bifurcation theory applied to symmetry groups. ${ }^{3}$ Besides, the development of the ring laser gyroscope has raised great practical interest, enhancing efforts toward a full understanding of the ring laser physics. ${ }^{4}$ More recently, semiconductor ring lasers ${ }^{5}$ (SRLs) have been demonstrated and studied because of their interesting features: They do not require cleaved facets or gratings for optical feedback, and thus monolithic integration is easily achievable. They are promising candidates for wavelength filtering, multiplexing-demultiplexing applications, electrical and all-optical switching, and bistable devices for optical memories. ${ }^{6}$ In this Letter we report, for the first time to our knowledge, the experimental observation of an oscillatory bidirectional regime in SRLs. Analytical expressions for oscillation threshold and frequency are calculated, and the $L-I$ curves are numerically reproduced with a two-mode model. A quantitative agreement between theory and measurements made over a large number of devices is obtained.

SRLs with $2-\mu \mathrm{m}$-wide single-transverse-mode ridge waveguides were fabricated in a double-quantum-well $\mathrm{GaAs} / \mathrm{AlGaAs}$ structure with a $1-\mathrm{mm}$ ring radius; the latter value was chosen to minimize bending losses. A sketch and a photographic detail of the device are shown in Fig. 1. An output straight waveguide with the same structure is directionally coupled to the ring, providing cross-power transmission of $\sim 10 \%$. The output waveguide has a $5^{\circ}$ tilt angle with respect to cleaved chip facets to minimize backreflections, and it terminates at each end in a reverse-biased contact that acts as an integrated photodetector for mode 1 (counterclockwise) and for mode 2 (clockwise). The reverse-biased contacts further reduce the optical feedback from the end facets. Figure 2(a) shows experimental $L-I$ curves measured at $25^{\circ} \mathrm{C}$ from the two integrated photodiodes, PD1 and PD2, as a function of dc current injected into the ring contact. The photocurrents from PD1 and PD2 are analyzed in their dc components, and they are also fed to a $50-\Omega$ digital oscilloscope and to a $\mathrm{rf}$ spectrum analyzer with which we investigate the dynamics. Three distinct operating regimes are identified, labeled $\mathrm{A}-\mathrm{C}$ in Fig. 2(a). In regime $\mathrm{A}$, which occurs from threshold (195 $\mathrm{mA}$ ) to $250 \mathrm{~mA}$, the ring laser operates bidirectionally; the intensities of the two counterpropagating modes are cw. Regime B, which occurs from 250 to $285 \mathrm{~mA}$, is again bidirectional, but in this case oscillatory behavior in the modal power is observed. The intensities of modes 1 and 2 are harmonically modulated at a frequency in the tens-of-megahertz range: When the intensity of mode 1 reaches a maximum, the intensity of mode 2 reaches a minimum, and vice versa. We call this regime alternate oscillations $(\mathrm{AO})$. In regime $\mathrm{C}$

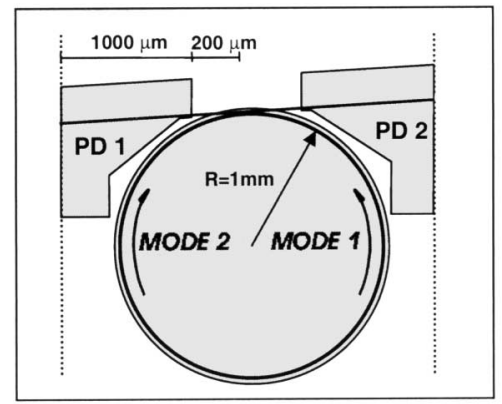

(a)

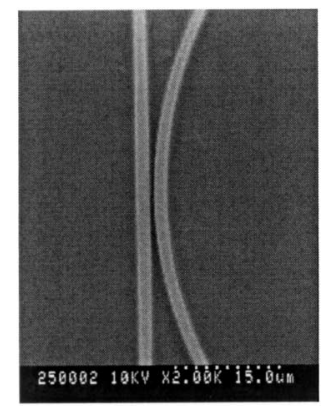

(b)
Fig. 1. (a) Geometry of the SRL device and layout of the contacts. PD1, PD2, photodiodes. (b) Detail of the output waveguide. 

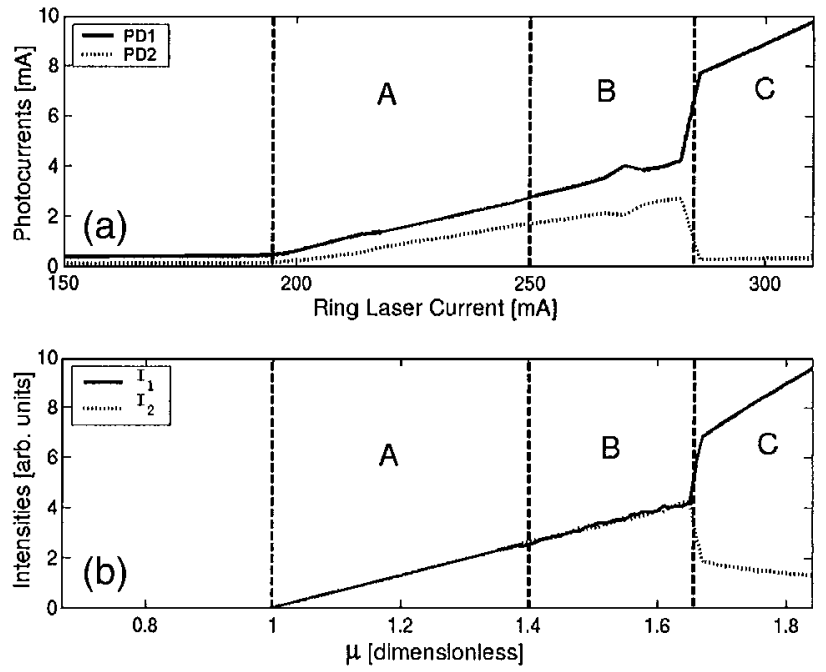

Fig. 2. (a) Experimental (dc) $L-I$ curves of a sample SRL device. (b) Theoretical time-averaged $L-I$ curve obtained with $\alpha=3.5, s=0.005, c=0.01, k_{c}=0.0044$ $k_{d}=0.000327, \gamma=0.002$, and $\tau_{p}=10 \mathrm{ps}$. The Hopf bifurcation boundary is $\mu_{H}=1.4$.

(above $285 \mathrm{~mA}$ ) the laser operates quasi-unidirectionally, and no oscillation occurs. When the current is further increased in regime $\mathrm{C}$, switching between the two modes is observed, as previously reported for SRLs. ${ }^{7}$ Optical spectrum measurements reveal that the SRL operates in a single longitudinal mode and that in bidirectional operation the wavelengths of the two counterpropagating modes are locked to the same value. Figure 3(a) reproduces oscilloscope traces of the intensities of modes 1 and 2 in regime $B$, showing AO. As the ring laser current is increased, the oscillation frequency decreases almost linearly, while the waveforms of the intensity oscillations become distorted into triangular shapes. Several SRLs from different batches were measured, and all devices exhibited the same behavior reported here. Regimes with bidirectional oscillatory instability were previously reported in gas $^{8}$ and dye ${ }^{9}$ ring lasers, but they were never observed in SRLs.

The theoretical analysis is based on a set of dimensionless semiclassical Lamb equations for the two (slowly varying) complex amplitudes of counterpropagating fields $E_{1}$ and $E_{2}$. The applicability of this theory to the devices that we fabricated is guaranteed by the single-transverse- and longitudinal-mode operation reported experimentally, by the absence of relevant feedback from outside the cavity and the reduced scattering loss achieved through the choice of a shallow-etched ridge structure and of a tilted and weakly coupled output waveguide. The Lamb equations read as

$$
\begin{aligned}
\dot{E}_{1,2}= & (1+i \alpha)\left[N\left(1-s\left|E_{1,2}\right|^{2}-c\left|E_{2,1}\right|^{2}\right)-1\right] \\
& \times E_{1,2}-\left(k_{d}+i k_{c}\right) E_{2,1},
\end{aligned}
$$

where $\alpha$ accounts for phase-amplitude coupling; the self- and cross-saturation coefficients are given by $s$ and $c$, and the parameters $k_{d}$ and $k_{c}$ represent the dissipative and the conservative components of the backscattering, respectively. ${ }^{8}$ Carrier density $N$ obeys the usual rate equation:

$$
\begin{aligned}
\dot{N}= & \gamma\left[\mu-N-N\left(1-s\left|E_{1}\right|^{2}-c\left|E_{2}\right|^{2}\right)\left|E_{1}\right|^{2}\right. \\
& \left.-N\left(1-s\left|E_{2}\right|^{2}-c\left|E_{1}\right|^{2}\right)\left|E_{2}\right|^{2}\right],
\end{aligned}
$$

where $\mu$ is the dimensionless pump ( $\mu=1$ at laser threshold). In Eqs. (1) and (2) the dimensionless time is rescaled by photon lifetime $\tau_{p}$. Parameter $\gamma$ is the ratio of photon lifetime $\tau_{p}$ to carrier lifetime $\tau_{s}$. The set of Eqs. (1) and (2) written for the intensities $S_{1,2}=\left|E_{1,2}\right|^{2}$ reverts to a previous model, ${ }^{10}$ except for inclusion of backscattering terms. In a real system the ideal symmetry along the ring is never met, for many reasons: imperfections in waveguide, output coupler, and scattering centers. Any break in the invariance symmetry along the ring translates into a source of coupling between the two counterpropagating fields. ${ }^{11}$ Thus backscattering terms have to be considered. In our approach, $k_{d}$ and $k_{c}$ are regarded as fitting parameters because their actual values are, in principle, technology dependent. According to a previous analysis, ${ }^{12}$ saturation parameters $c$ and $s$ in a SRL fulfill the condition that $c / s>1$.

By substituting the general solution $E_{1,2}=$ $Q_{1,2}(t) \exp \left[i \omega_{1,2} t+i \phi_{1,2}(t)\right]$ into Eqs. (1) and (2) we find the steady-state solutions by setting all the derivatives to zero. We find a symmetric $\left(Q_{1}=Q_{2}=Q\right)$ steady state (SS). When we introduce $\psi=\phi_{2}-\phi_{1}$ and $I=2 Q^{2}, \mathrm{SS}=\left(\omega_{1,2}, \psi, I_{0}, N_{0}\right)$ is given by $\omega_{1}=\omega_{2}=-\alpha k_{d}+k_{c}, \psi_{0}=\pi$ :

$$
\begin{aligned}
I_{0} & =\frac{N_{0}-1+k_{d}}{\eta N_{0}}, \\
N_{0} & =\frac{\mu}{1+I_{0}-\eta I_{0}^{2}},
\end{aligned}
$$

where $\eta=(c+s) / 2$. After linearization of the perturbations defined by $E_{1,2}=\left(\sqrt{I_{0} / 2}+\right.$ $\left.a_{1,2}\right) \exp \left(i \omega_{1,2} t+i \phi_{1,2}\right)$ and $N=N_{0}+\Delta$, a linear stability analysis of solutions [Eqs. (3) and (4)] is performed ${ }^{13} ; a_{1,2}$ are complex perturbations of the field amplitudes, and $\Delta$ is a real perturbation of the carrier variables. To first order in the perturbations,

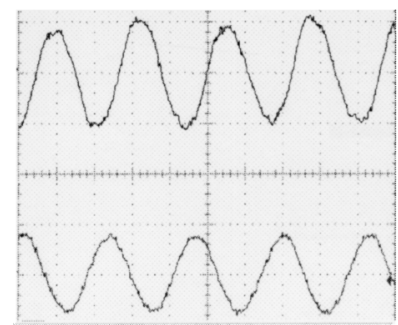

(a)

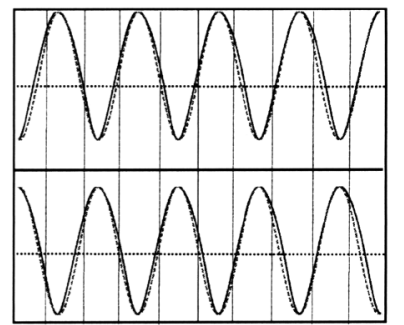

(b)
Fig. 3. Time traces of the intensities of modes 1 and 2 in the AO regime. (a) Experimental single-shot acquisition measured from PD1 and PD2 on a $50-\Omega$ oscilloscope: vertical scale, $50 \mathrm{mV} /$ division; horizontal scale, $10 \mathrm{~ns} /$ division; ring current, $275 \mathrm{~mA}$. (b) Result obtained through numerical simulations (solid curves) and from application of Eq. (8) (dashed curves): vertical scale, arbitrary units; horizontal scale, $10 \mathrm{~ns} /$ division. The parameters are the same as for Fig. 2 , and $\mu=1.58$. 
the system of Eqs. (1) and (2) decouples into two subsets. The first subset accounts for the total intensity stability; it contains the variables $S=a_{2}+a_{1}$ and $\Delta$, and it is always stable. The second subset contains the complex variable $R=a_{2}-a_{1}$ and describes the stability of one field with respect to the other:

$$
\begin{aligned}
\dot{R}= & 1 / 2(1+i \alpha) N_{0} I_{0}(c-s)\left(R+R^{*}\right) \\
& -2\left(k_{d}+i k_{c}\right) R \\
\dot{R}^{*}= & 1 / 2(1-i \alpha) N_{0} I_{0}(c-s)\left(R+R^{*}\right) \\
& -2\left(k_{d}-i k_{c}\right) R^{*} .
\end{aligned}
$$

The calculation of the eigenvalues associated with Eqs. (5) and (6) shows that near threshold the SS is stable. As the pump is increased, the SS loses stability through a Hopf bifurcation when

$$
4 k_{d}=N_{0} I_{0}(c-s),
$$

exhibiting pulsating behavior at the frequency

$$
\Omega=2\left[k_{d}{ }^{2}+k_{c}{ }^{2}-\frac{N_{0} I_{0}}{2}\left(k_{d}+\alpha k_{c}\right)\right]^{1 / 2} .
$$

We remark that an in-phase solution $(\psi=0)$ also exists, but it is always unstable and we do not consider it here. When $I_{0}$ and $N_{0}$ are expressed as functions of $\mu$, a particular value of $\mu=\mu_{H}$ satisfies Eq. (7). Thus, for $1<\mu<\mu_{H}$, bidirectional stable operation is predicted; when $\mu>\mu_{H}$, oscillatory behavior takes place. This oscillation represents a limit cycle on the variables $R$ and $R^{*}$ and corresponds to AO of the two mode intensities $\left|E_{1,2}\right|^{2}$. The AO angular frequency at onset is obtained by substitution of Eq. (7) into Eq. (8), yielding $\Omega_{H}=\left(k_{c}{ }^{2}-k_{d}{ }^{2}+2 \alpha k_{d} k_{c}\right)^{1 / 2}$. When the pump is increased further, the oscillation frequency decreases, until oscillations disappear when two new quasiunidirectional solutions become stable. In that case the output power is concentrated primarily in one direction, and no pulsation occurs. The analytical description of this transition is rather involved and will be the subject of further investigations.

Figures 2(b) and 3(b) illustrate numerical results obtained by Eqs. (1) and (2) through a standard Runge-Kutta algorithm. We found that the pump interval for the $\mathrm{AO}$ regime widens when $k_{c}$ is increased and shrinks when $k_{d}$ is increased. The observed SRL dynamics can be interpreted as follows: Because of the strong cross-gain saturation, the semiconductor medium tends to select unidirectional operation.
However, because of backscattering, the pure unidirectional state is not a solution for the ring cavity, and bidirectional regimes are favored. The tendency to unidirectional behavior is recovered at a higher pump level, at which nonlinear gain imposes a stronger mode selection. The AO regime reported here has some analogies to the $\mathrm{AO}$ induced by conservative backscattering in $\mathrm{He}-\mathrm{Ne}$ ring lasers. ${ }^{8}$ However, an important difference lies in the fact that the $\mathrm{He}-\mathrm{Ne}$ ring laser supports bidirectional operation (clockwise and counterclockwise waves carry the same amount of energy during the pulsation) because $c / s<1$.

In conclusion, we have fabricated and tested single-longitudinal- and transverse-mode semiconductor ring lasers. A bifurcation from bidirectional stable operation to oscillatory behavior has been measured. and it was described through a two-mode approach, yielding analytical expressions for the oscillations' onset and frequency near the bifurcation; the whole experimental $L-I$ curve is well reproduced by theory.

The authors acknowledge technical support from the Nanoelectronics Research Center and the dry etching group of the Department of Electronics and Electrical Engineering, Glasgow University, and from A. Fanzio. A. Scirè thanks the European Union for the financial support of a Marie Curie fellowship under contract HPMF-CT-2000-00617. A. Scirè's e-mail address is scire@imedea.uib.es.

\section{References}

1. C. O. Weiss and R. Vilaseca, Dynamics of Lasers (Weinheim, New York, 1991), and references therein.

2. Q. L. Williams and R. Roy, Opt. Lett. 37, 470 (1988).

3. E. J. D'Angelo, E. Izaguirre, G. B. Mindlin, L. Gil, and J. R. Tredicce, Phys. Rev. Lett. 68, 3702 (1992).

4. W. W. Chow, J. Gea-Banacloche, L. M. Pedrotti, V. E. Sanders, W. Schleich, and M. O. Scully, Rev. Mod. Phys. 57, 61 (1985).

5. T. Krauss, P. J. R. Laybourn, and J. S. Roberts, Electron. Lett. 26, 2095 (1990).

6. J. J. Liang, S. T. Lau, M. H. Leary, and J. M. Ballantyne, Appl. Phys. Lett. 70, 1192 (1997).

7. M. Sorel, G. Giuliani, S. Donati, and P. J. R. Laybourn, Appl. Phys. Lett. 80, 3051 (2002).

8. R. J. C. Spreew, R. Centeno Neelon, N. J. van Druten, E. R. Eliel, and J. P. Woerdman, Phys. Rev. A 42, 4315 (1990).

9. F. C. Cheng, Phys. Rev. A 45, 5220 (1992).

10. T. Numai, IEEE J. Quantum Electron. 36, 1161 (2000).

11. M. San Miguel, Phys. Rev. Lett. 75, 425 (1995).

12. M. Sargent, Phys. Rev. A 48, 717 (1993).

13. C. Etrich, P. Mandel, R. Centeno Neelen, R. J. C. Spreeuw, and J. P. Woerdman, Phys. Rev. A 46, 525 (1992). 